\title{
Autonomic dysfunction in patients with nocturnal hypoventilation in extrapulmonary restrictive disease
}

\author{
J.P. Watson*, J. Nolan**, M.W. Elliott ${ }^{+}$
}

Autonomic dysfunction in patients with nocturnal hypoventilation in extrapulmonary restrictive disease. J.P. Watson, J. Nolan, M.W. Elliott. C) ERS Journals Ltd 1999.

ABSTRACT: In chronic obstructive pulmonary disease, persistent hypoxia may be associated with autonomic dysfunction. The effect of nocturnal oxygen desaturation on autonomic function in patients with chest wall deformities and neuromuscular disease is unknown. This study examined the effect of nocturnal oxygen desaturation upon heart rate variability, a sensitive measure of autonomic function.

Twenty-seven patients with chest wall deformity or neuromuscular disease underwent analysis of overnight oximetry, blood gases, and $24 \mathrm{~h}$ heart rate variability (HRV), specifically the standard deviation of normal-to-normal (SDNN) RR intervals, and the number of increases in successive $N N$ intervals $>50$ ms (sNN50). Subjects were grouped according to nocturnal arterial oxygen saturation $\left(\mathrm{S}_{\mathrm{a}}, \mathrm{O}_{2}\right)$ : group $1 \mathrm{had}$ episodes of $\mathrm{Sa}_{\mathrm{a}} \mathrm{O}_{2}<90 \%$, group 2 had $\mathrm{Sa}_{\mathrm{a}} \mathrm{O}_{2}>90 \%$ throughout the night, and group 3 were 27 healthy age-matched controls who also underwent HRV analysis.

The mean \pm SD SDNN for group 1 was $79.3 \pm 23.7 \mathrm{~ms}$, less than group $2(149.8 \pm 58.9 \mathrm{~ms}$, $p<0.02)$ and group $3(155.1 \pm 37.1 \mathrm{~ms}, \mathbf{p}<0.001)$. The geometric mean sNN50 was less in group 1 than group $2(1,530$ versus $5,843, p<0.01)$, but not significantly different from group $3(2,712, p=0.053)$. There was no significant difference between groups 2 and 3 . Within group 1, both SDNN and sNN50 were significantly lower in those patients with more severe nocturnal hypoxia. The minimum overnight $S_{\mathrm{a}}, \mathrm{O}_{2}$ was the best predictor of abnormal HRV.

In conclusion, patients with nocturnal hypoxia have evidence of autonomic dysfunction, even in cases with only transient episodes of nocturnal oxygen desaturation. The severity of autonomic dysfunction is related to the degree of nocturnal oxygen desaturation.

Eur Respir J 1999; 13: 1097-1102.

Depts of *Respiratory Medicine, and **Cardiology, Leeds General Infirmary, Leeds, UK. ${ }^{+}$Dept of Respiratory Medicine, St James' University Hospital, Leeds, UK.

Correspondence: J.P. Watson

Dept of Respiratory Medicine

Leeds General Infirmary

Great George Street

Leeds LS1 3EX

UK

Fax: 441133926316

Keywords: Autonomic dysfunction heart rate variability

hypoventilation

hypoxaernia

kyphoscoliosis

Received: June 301998

Accepted after revision January 131999
Patients with chest wall deformity or neuromuscular disease may develop nocturnal hypoventilation in the absence of significant pulmonary disease. Noninvasive ventilation is effective in treating diurnal ventilatory failure owing to nocturnal hypoventilation in such patients $[1,2]$. It is usually initiated when patients become symptomatic, by which time the nocturnal derangement of gas exchange is severe. At an earlier stage in the natural history of the condition, patients may have oxygen desaturation during sleep but a relatively normal daytime arterial oxygen tension $\left(\mathrm{Pa}_{\mathrm{a}} \mathrm{O}_{2}\right)$. Chronic hypoxia is associated with significant morbidity and mortality. However, it is not known whether asymptomatic nocturnal hypoxia in these patients is harmful, and if so, what degree of nocturnal oxygen desaturation is damaging. Similarly, it is not known whether there is any difference in the effect of modest desaturation occurring throughout the night compared with a relatively normal saturation for most of the time but with occasional episodes of severe desaturation, such as that seen in rapid eye movement sleep [3].

In patients with persistent hypoxia due to chronic obstructive pulmonary disease (COPD), survival is improved by long-term oxygen therapy (LTOT) $[4,5]$. It is unclear what degree or duration of hypoxia is harmful, although for LTOT to be effective a minimum treatment period of $15 \mathrm{~h}$ daily is recommended. The mechanisms mediating the reduction in mortality are unclear, but it has been suggested that patients with COPD may be at risk of sudden deaths due to arrhythmias [6]. There is evidence of an increased prevalence of autonomic dysfunction, based on cardiovascular reflex testing [7, 8], acetylcholine sweat-spot scores [9], and studies of electrocardiographic QTc intervals [10] in COPD. Autonomic dysfunction in other conditions is associated with myocardial electrical instability and a high risk of arrhythmic death, and in COPD patients is associated with an increased mortality [10]. Disordered peripheral nerve function is not uncommon in patients with COPD; KINSMAN et al. [11] found subjective sensory symptoms in $40 \%$ of 146 patients with COPD, and the peripheral nerve involvement was correlated with the severity of the chest disease. Possible aetiological factors for peripheral neuropathy in COPD include hypoxia, cigarette smoking, alcohol and malnutrition [12]. It is therefore possible that hypoxia causes the autonomic dysfunction seen in COPD, and this may contribute to the excess mortality in the patients with untreated hypoxia. The presence of autonomic dysfunction may therefore be a useful early marker of the adverse effects of hypoxia. 
The effect of nocturnal oxygen desaturation on autonomic nervous function was studied in patients with chest wall deformities and neuromuscular disease, and the effects of different degrees of nocturnal oxygen desaturation compared.

\section{Methods}

\section{Subjects}

Patients with kyphoscoliosis, other musculoskeletal disorders or neuromuscular conditions which may cause hypoventilation, presenting to a regional noninvasive ventilation unit were invited to take part in the study. Patients with evidence of recent myocardial infarction, diabetes mellitus or other conditions known to be independently associated with autonomic neuropathy were excluded to avoid confounding effects. Patients with obstructive sleep apnoea or cardiac dysrhythmias, which could directly interfere with heart rate variability (HRV) analysis, were also excluded. A total of 31 subjects were invited to participate, of whom 30 gave written informed consent. Three subjects were subsequently excluded because they were found to have an arrhythmia which precluded HRV analysis, leaving 27 subjects for analysis. Eight subjects were taking inhaled $\beta$-agonists (salbutamol "as required" in doses up to $800 \mu \mathrm{g}$ daily), and four were on protriptyline (10 mg daily). No patients had been treated with LTOT and none were on $\beta$ blockers. In addition, 27 age-matched healthy subjects, with no history of diabetes, obstructive sleep apnoea, cardiac or respiratory disease, were studied as controls. No controls were taking any medications. The study was approved by the research ethical committee of the United Leeds Teaching Hospitals National Health Service Trust.

\section{Data collection}

Each subject had daytime arterial blood gases measured while breathing room air at rest. Forced expiratory volume in one second (FEV1) and forced vital capacity (FVC) were measured using a Vitalograph ${ }^{\mathrm{TM}}$ spirometer (Vitalograph Ltd., Buckingham, UK). Oxygen saturation was recorded continuously throughout one night of breathing air without ventilatory support, using an Ohmeda Biox $3740^{\mathrm{TM}}$ Pulse Oximeter (Ohmeda, Louisville, CO, USA). The recording was subsequently downloaded into a computer and the mean, median, and minimum oxygen saturations were calculated, as well as the total duration for which oxygen saturation was $<90 \%$.

\section{Heart rate variability analysis}

Autonomic function was assessed using HRV analysis. This is a simple, sensitive, and specific test [13], which is reproducible in normal and abnormal subjects [14], and correlates well with other markers of autonomic dysfunction, including clinical tests of cardiovascular reflexes [15]. Twenty-four hour ambulatory electrocardiograms were obtained for each subject using a miniature tape recorder (Tracker II ${ }^{\mathrm{TM}}$, Reynolds Medical Ltd., Hertford, UK) with a crystal-generated timing track that allows correction for recording and replay speed errors and has some practical advantages over other recording systems [16].
The recordings were replayed through a Pathfinder arrhythmia analyser (Reynolds Medical Ltd). Segments of tape in which changes in the RR interval arise due to supraventricular or ventricular ectopics were excluded from analysis by the analyser, which can detect such ectopics by their difference in timing and morphology [17]. Because the analyser takes no account of P-wave morphology, and is thus unable to determine whether complexes showing minor degrees of prematurity are of sinus origin, the signal was closely monitored by experienced operators, who discarded segments of tape in which frequent late coupled supraventricular complexes of possible ectopic origin occurred. The overall accuracy of QRS detection with this system is high [18], and the speed surveyor in the replay unit minimizes inaccuracy due to speed variation. The system is therefore ideal for HRV measurements [16]. The remaining normal-to-normal (NN) RR intervals were measured, and the standard deviation of these intervals ( $\mathrm{SDNN}$ ) in milliseconds was calculated. This is influenced by changes in both sympathetic and parasympathetic activity and so reflects sympathovagal balance [19]. Increases in successive NN intervals $>50 \mathrm{~ms}$ (sNN50) were counted as previously described $[20,21]$. To facilitate comparison between subjects, counts were normalized to the exact $24 \mathrm{hr}$ value and this standardized value was presented as the sNN50 count, which is principally influenced by changes in parasympathetic activity [20]. The operators performing the HRV analysis were blind to the oximetry results.

\section{Statistical analysis}

Since sNN50 counts are not normally distributed [20], the values were $\log$ transformed prior to statistical analysis. Group data for $\mathrm{sNN} 50$ are presented as geometric mean (range). Groups were compared using Student's ttests for SDNN and for $\log _{10} \mathrm{sNN} 50$, and correlations were analysed by the Pearson's product-moment correlation. A p-value $<0.05$ was regarded as significant.

\section{Results}

Subjects were divided into groups depending on the overnight oximetry results. Group 1 had episodes of nocturnal oxygen desaturation (oxygen saturation $<90 \%$ at any time during overnight recording), group 2 had no episodes of oxygen desaturation, with oxygen saturation remaining $>90 \%$ throughout the recording period, and group 3 comprised the healthy controls. Group 1 was arbitrarily divided into subgroups based on the severity of nocturnal oxygen desaturation. Subgroup la was defined by a median nocturnal oxygen saturation $<90 \%$, which implies prolonged hypoxaemia. Subgroup $1 \mathrm{~b}$ had more transient episodes of oxygen desaturation giving a median oxygen saturation $>90 \%$, so they were not significantly hypoxic for most of the night.

In group 1 there were 21 subjects ( 12 male), aged 24-72 yrs (median 60 yrs), of whom 12 were in subgroup 1a and nine were in subgroup 1 b. Group 2 included six subjects (four male) aged 16-65 yrs (median 45 yrs). Group 3 comprised 27 healthy controls (24 male) aged 18-65 yrs (median 54 yrs). Detailed information about the subjects is given in tables 1 and 2, and the underlying diagnoses are listed in table 3. 
Table 1. - Physical characteristics of subjects in the two groups, including the two subgroups of group 1

\begin{tabular}{|c|c|c|c|c|}
\hline & \multicolumn{4}{|c|}{ Group } \\
\hline & 1 & $1 \mathrm{a}$ & $1 \mathrm{~b}$ & 2 \\
\hline Sex M/F & $12 / 9$ & $8 / 4$ & $4 / 5$ & $4 / 2$ \\
\hline Age yrs & $54.4 \pm 13.7$ & $56.8 \pm 12.4$ & $51.2 \pm 15.4$ & $41.2 \pm 18.7$ \\
\hline \multicolumn{5}{|l|}{ Smokers } \\
\hline Current & 3 & 2 & 1 & 0 \\
\hline Ex & 3 & 1 & 2 & 1 \\
\hline BMI $\mathrm{kg} \cdot \mathrm{m}^{-2}$ & $24.2 \pm 3.1$ & $24.4 \pm 3.4$ & $23.7 \pm 2.7$ & $22 \pm 6.0$ \\
\hline FEV1 L & $0.76 \pm 0.32$ & $0.72 \pm 0.34$ & $0.81 \pm 0.30$ & $1.55 \pm 1.16$ \\
\hline FVC L & $0.96 \pm 0.39$ & $0.93 \pm 0.40$ & $1.01 \pm 0.40$ & $2.04 \pm 1.48$ \\
\hline
\end{tabular}

Data are presented as mean \pm SD. Group 1: episodes of nocturnal oxygen desaturation (oxygen saturation $<90 \%$ at any time during overnight recording); Group 1a: median nocturnal oxygen saturation $<90 \%$, implying prolonged hypoxaemia; Group $1 \mathrm{~b}$ : transient episodes of oxygen desaturation giving a median oxygen saturation $>90 \%$, i.e. not significantly hypoxic; Group 2 : no episodes of oxygen desaturation, with oxygen saturation remaining $>90 \%$. M: male; F: female; BMI: body mass index; FEV1: forced expiratory volume in one second; FVC: forced vital capacity.

The SDNN results are shown in figure 1 . The mean \pm SD SDNN value for group 1 was $79.3 \pm 23.7 \mathrm{~ms}$ which was significantly less than for both group $2(149.8 \pm 58.9 \mathrm{~ms}$, $\mathrm{p}<0.02)$, and group $3(155.1 \pm 37.1 \mathrm{~ms}, \mathrm{p}<0.001)$. There was no significant difference between groups 2 and 3 . The sNN50 results are shown in figure 2. The geometric mean was significantly less in group 1 than in group 2 : 1,530 and 5,843 , respectively $(\mathrm{p}<0.01)$. However, the difference between groups 1 and 3 (sNN50 geometric mean 2,712$)$ was not statistically significant $(\mathrm{p}=0.053)$. The difference between groups 2 and 3 was not significant.

There are no clearly defined "normal" values for SDNN or sNN50. Based on a sample of 57 healthy males, the lower $95 \%$ confidence limit for sNN50 has been estimated as 1,000 at $45 \mathrm{yrs}$ of age and 500 at $65 \mathrm{yrs}$ of age [22]. Using these values, five subjects in group 1 , none in group 2 and two in group 3 had sNN50 below the minimum 95\% confidence limit for their age. An SDNN $<100$ $\mathrm{ms}$ has been shown to be associated with a high mortality in chronic heart failure [23]. Seventeen subjects in group 1 , one in group 2, and one in group 3 had SDNN values $<100 \mathrm{~ms}$.

When the milder and more severely hypoxic subgroups were compared, mean \pm SD SDNN was $69.6 \pm 21.8 \mathrm{~ms}$ for subgroup $1 \mathrm{a}$ and $92.2 \pm 20.6 \mathrm{~ms}$ for subgroup $1 \mathrm{~b}(\mathrm{p}<0.02)$.
Table 3. - Underlying disease diagnosis in the two main groups and subgroups of group 1

\begin{tabular}{lrccc}
\hline & \multicolumn{5}{c}{ Group } \\
\cline { 2 - 5 } Diagnosis & 1 & $1 \mathrm{a}$ & $1 \mathrm{~b}$ & 2 \\
$(\mathrm{n}=21)$ & $(\mathrm{n}=12)$ & $(\mathrm{n}=9)$ & $(\mathrm{n}=6)$ \\
\hline $\begin{array}{l}\text { Early onset kyphoscoliosis } \\
\text { Thoracoplasty }\end{array}$ & 14 & 9 & 5 & 2 \\
$\begin{array}{l}\text { Potts kyphosis } \\
\text { Muscular dystrophy }\end{array}$ & 1 & 2 & & 1 \\
$\begin{array}{l}\text { Neurofibromatosis } \\
\text { Hereditary motor neuropathy }\end{array}$ & 2 & 1 & 1 & 2 \\
Motor neurone disease & 2 & & 2 & 1 \\
\hline
\end{tabular}

Groups are as defined in footnote to table 1 .

The geometric mean of sNN50 was 911 for subgroup 1a and 3,053 for subgroup $1 b(p<0.02)$. The sDNN for the less severely hypoxic subgroup $1 \mathrm{~b}$ was still significantly lower than for group $2(\mathrm{p}<0.04)$.

Combining groups 1 and 2, there were significant correlations with the minimum overnight oxygen saturation for both $\operatorname{sDNN}(\mathrm{r}=0.62, \mathrm{p}<0.001)$ and $\log _{10} \mathrm{sNN} 50(\mathrm{r}=0.52$, $\mathrm{p}<0.01$ ) (figs. 3 and 4). There was also a significant but weaker correlation between SDNN and median overnight oxygen saturation $(\mathrm{r}=0.41, \mathrm{p}<0.05)$, and within group 1 , there was an inverse relationship between SDNN and the total duration for which nocturnal oxygen saturation was $<90 \%(\mathrm{r}=-0.44, \mathrm{p}<0.05)$. Group 2 was excluded from the latter analysis because by definition the duration of oxygen saturation $<90 \%$ was zero. There was no significant relationship between $\log _{10} \mathrm{sNN} 50$ and either the median saturation or the duration for which oxygen saturation was $<90 \%$.

The mean \pm SD daytime $P \mathrm{a}, \mathrm{O}_{2}$ was $8.8 \pm 1.9 \mathrm{kPa}$ in group 1 and $12.4 \pm 1.8 \mathrm{kPa}$ in group $2(\mathrm{p}<0.01)$. Five subjects in group 1 had a $P \mathrm{a}_{2} \mathrm{O}_{2}<7.3 \mathrm{kPa}$, the threshold for LTOT in COPD. Combining the results from all subjects in groups 1 and 2 , there were moderate but statistically significant correlations between SDNN and daytime $\mathrm{Pa}_{\mathrm{a}} \mathrm{O}_{2}(\mathrm{r}=0.52$, $\mathrm{p}<0.01)$ and arterial carbon dioxide tension $\left(P \mathrm{a}, \mathrm{CO}_{2}\right)(\mathrm{r}=$ $-0.50, \mathrm{p}<0.01)$. Similarly, there were correlations between $\log _{10}$ sNN50 and $\mathrm{Pa}_{\mathrm{a}} \mathrm{O}_{2}(\mathrm{r}=0.50, \mathrm{p}<0.01)$ and $\mathrm{Pa}_{\mathrm{a}} \mathrm{CO}_{2}(\mathrm{r}=$ $-0.40, \mathrm{p}<0.05)$.

The mean \pm SD FVC was $0.96 \pm 0.39 \mathrm{~L}$ in group 1 , and $2.04 \pm 1.48 \mathrm{~L}$ in group 2 . This difference was not statistically significant. There was a moderate correlation between SDNN and FVC ( $\mathrm{r}=0.46, \mathrm{p}<0.02)$, but no significant relationship between $\log _{10} \mathrm{sNN} 50$ and $\mathrm{FVC}(\mathrm{r}=0.35, \mathrm{p}>$ $0.05)$.

Table 2. - Mean \pm SD oximetry and blood gas data for the two groups and subgroups

\begin{tabular}{lcccc}
\hline & \multicolumn{4}{c}{ Group } \\
\cline { 2 - 5 } & 1 & $1 \mathrm{a}$ & $1 \mathrm{~b}$ & 2 \\
\hline Median overnight $\mathrm{Sa}_{\mathrm{a}, \mathrm{O}_{2} \%}$ & $86.9 \pm 10.0$ & $81.3 \pm 9.92$ & $94.2 \pm 2.8$ & $96.0 \pm 1.4$ \\
Time with $\mathrm{a}_{\mathrm{a}, \mathrm{O}_{2}<90 \% \text { min }}$ & $246.9 \pm 191.8$ & $393.1 \pm 99.7$ & $52 \pm 59.7$ & $0.0 \pm 0.0$ \\
Minimum overnight $\mathrm{Sa}_{2} \mathrm{O}_{2} \%$ & $69.1 \pm 11.8$ & $63.6 \pm 11.5$ & $76.6 \pm 7.5$ & $92.0 \pm 2.9$ \\
$\mathrm{pH}$ & $7.39 \pm 0.03$ & $7.38 \pm 0.02$ & $7.4 \pm 0.03$ & $7.42 \pm 0.07$ \\
$P \mathrm{a}, \mathrm{CO}_{2} \mathrm{kPa}$ & $6.5 \pm 1.1$ & $7.0 \pm 0.8$ & $5.8 \pm 1.1$ & $5.1 \pm 0.4$ \\
$\mathrm{~Pa}_{\mathrm{a}} \mathrm{KPa}$ & $8.8 \pm 1.9$ & $7.6 \pm 0.9$ & $10.4 \pm 1.7$ & $12.4 \pm 1.8$ \\
\hline
\end{tabular}

Data are presented as mean \pm SD. $S \mathrm{a}, \mathrm{O}_{2}$ : arterial oxygen saturation; $P_{\mathrm{a}, \mathrm{CO}_{2}}$ : arterial carbon dioxide tension; $P \mathrm{a}, \mathrm{O}_{2}$ : arterial oxygen tension. Groups are as defined in footnote to table 1 . 


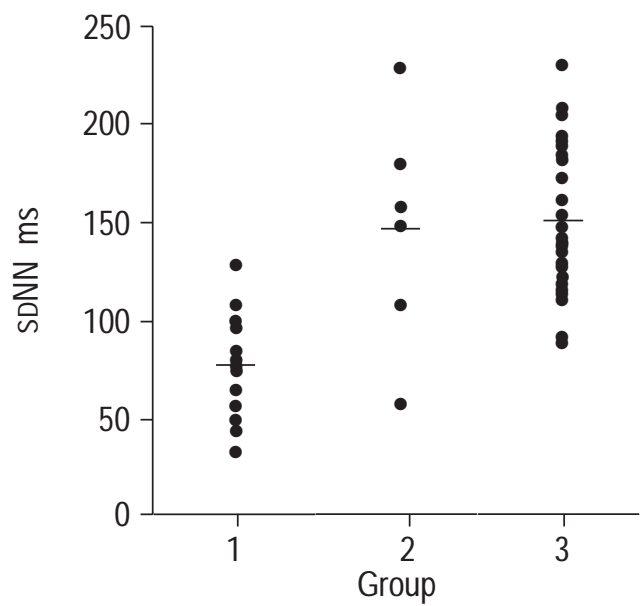

Fig. 1. - Standard deviation of the normal-to-normal (SDNN) RR interval values for subjects in each group. Group 1 and 2 as defined in footnote to table 1 and group 3 are healthy age-matched control. Horizontal bars represent the group mean.

There was no correlation between SDNN and age, but there was a decline in $\log _{10} \mathrm{sNN} 50$ with increasing age $(\mathrm{r}=$ $-0.41, \mathrm{p}=0.002$ ). However, the group 3 controls were age matched to the cases, and there was no significant difference in age between groups 1 and 2, or between the group 1 subgroups.

There was an inverse relationship between SDNN and 24 $\mathrm{h}$ mean heart rate (MHR; $\mathrm{r}=-0.65, \mathrm{p}<0.001)$. The mean $\pm \mathrm{SD}$ MHR was faster in group $1\left(90.0 \pm 11.4\right.$ beats. $\left.\min ^{-1}\right)$, than group $2\left(78.2 \pm 12.8\right.$ beats $\left.\mathrm{min}^{-1} ; \mathrm{p}=0.04\right)$. However, there was no difference in MHR between subgroup 1a (90.8 \pm 11.1 beats $\left.\cdot \mathrm{min}^{-1}\right)$ and subgroup $1 \mathrm{~b}\left(89.0 \pm 12.5\right.$ beats $\left.\cdot \mathrm{min}^{-1}\right)$. There was no correlation between $\mathrm{SNN} 50$ and heart rate.

Although several of the variables detailed above were found to be correlated with SDNN, $\log _{10} \mathrm{SNN} 50$, or both, these variables were not independent of each other. All these variables were therefore entered into multiple regression analysis. For both $\mathrm{SDNN}$ and $\log _{10} \mathrm{sNN} 50$, minimum overnight oxygen saturation was the only variable to remain independently significantly correlated.

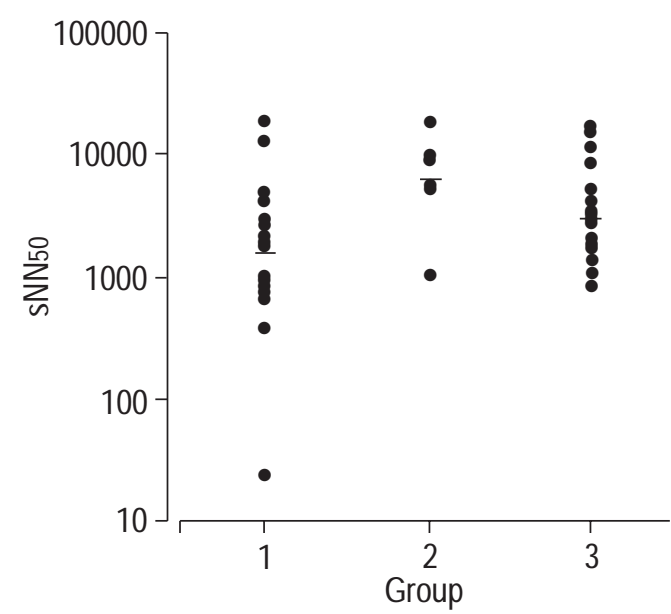

Fig. 2. - Successive normal-to-normal interval $>50 \mathrm{~ms}$ (sNN50) values for subjects in each group. Groups as defined in figure 1 . Horizontal bars represent the group geometric mean.

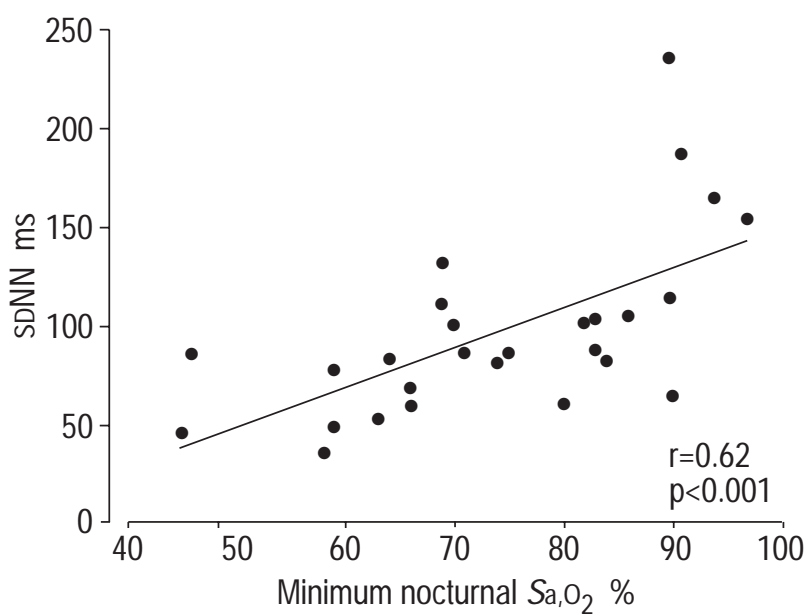

Fig. 3. - Relationship between the standard deviation of the normal-tonormal (SDNN) RR intervals and minimum nocturnal arterial oxygen saturation $\left(\mathrm{S}_{\mathrm{a}}, \mathrm{O}_{2}\right)$ for groups 1 and 2 combined (see table 1 footnote for definitions).

When patients with neurological disorders, rather than purely mechanical chest wall disorders were excluded from the analysis, the results were essentially unchanged. In group 1 , the mean \pm SD SDNN was $79.8 \pm 25.9 \mathrm{~ms}$, significantly less than group $3(\mathrm{p}<0.001)$. The geometric mean of sNN50 was 1,144, significantly less than in group $3(\mathrm{p}<0.02)$. The SDNN in subgroup 1a was significantly less than subgroup $1 \mathrm{~b}$. There were too few patients left in group 2 for comparison.

\section{Discussion}

This study has demonstrated that patients with nocturnal oxygen desaturation due to musculoskeletal chest wall abnormalities have significant abnormalities of HRV, which indicates autonomic dysfunction. The differences between subgroups $1 \mathrm{a}$ and $1 \mathrm{~b}$ suggests that severity of autonomic dysfunction appears to be related to the severity

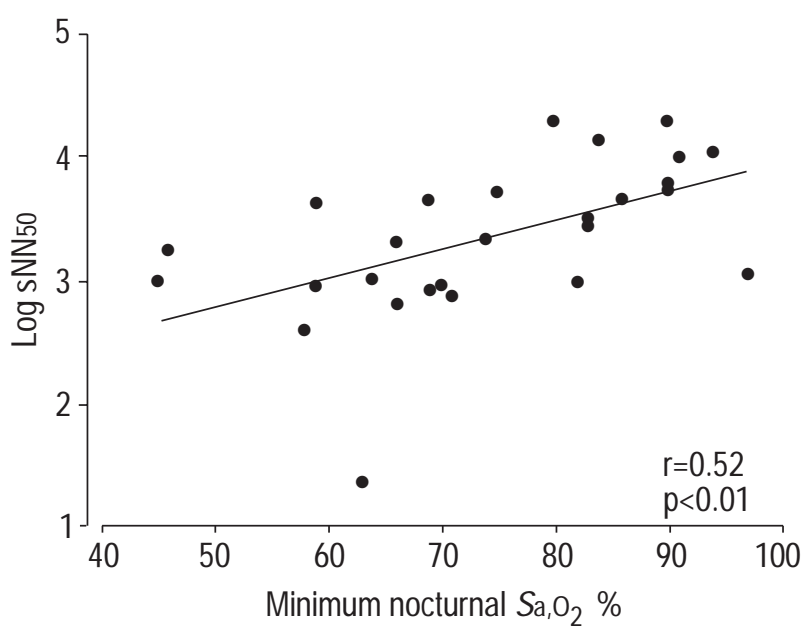

Fig. 4. - Relationship between $\log _{10}$ successive normal-to-normal intervals $>50 \mathrm{~ms}$ ( $\mathrm{sNN} 50$ ) and minimum nocturnal arterial oxygen saturation $\left(\mathrm{S}_{\mathrm{a}, \mathrm{O}_{2}}\right)$ for groups 1 and 2 combined (see table 1 footnote for definitions). 
of oxygen desaturation, but even those with less severe nocturnal oxygen desaturation had significant abnormalities compared to those with no oxygen desaturation. The strongest predictor of both $\mathrm{SDNN}$ and $\mathrm{SNN} 50$ was the minimum level to which oxygen saturation fell during the night, which gave a stronger correlation than either the duration of hypoxia or the median nocturnal oxygen saturation, supporting the conclusion that even transient episodes of desaturation may be important.

Those patients with the most severe nocturnal oxygen desaturation, also tended to have a lower daytime $P \mathrm{a}, \mathrm{O}_{2}$ and a higher $P \mathrm{a}, \mathrm{CO}_{2}$, and there were correlations between HRV variables and daytime blood gases. It is unclear to what extent the HRV abnormalities may be related to moderate daytime blood gas abnormalities as well as nocturnal hypoventilation. However, the correlations were closer for minimum nocturnal oxygen saturation, and in multiple regression analysis, neither daytime $\mathrm{Pa}, \mathrm{CO}_{2}$ nor $\mathrm{Pa}, \mathrm{O}_{2}$ were independently correlated with SDNN or $\log _{10} \mathrm{sNN} 50$. In subgroup $1 \mathrm{~b}$, sDNN was low, but the mean daytime $P \mathrm{a}, \mathrm{O}_{2}$ was near normal.

A decline in sNN50 with age has been reported previously in a healthy population [22]. The same study showed no difference in sex, so although it would have been preferable to recruit group 3 controls matched for sex in the present study, it was felt that age matching was more important. There was no significant difference between the subject groups or subgroups in the male/ female distribution. Smoking has been reported to reduce vagal tone [24]. However, only three of the 27 patients were current smokers so this is unlikely to have significantly influenced the results of this study.

One component of normal HRV is the parasympathetic response to changes in intrathoracic pressure during respiration. It is possible that for patients in this study, reduced chest wall movements may have contributed to a reduced parasympathetic response even if autonomic functions were normal. This could affect the sNN50 results, but the SDNN index is modulated by multiple influences, several of which are not related to respiration. There was no correlation between $\log _{10} \mathrm{sNN} 50$ and FVC, and in multiple regression analysis the relationship between SDNN and FVC was not independent of minimum oxygen saturation, suggesting that differences in chest wall movements alone cannot explain the abnormalities found.

It is possible that some neurological diseases may be associated with primary cardiac or autonomic abnormalities but the authors' know of no evidence that the conditions of the present subjects are associated with abnormal HRV. Excluding subjects with neurological disorders from the analysis made no difference to the results for SDNN, and the difference between groups 1 and 3 for sNN50 became statistically significant. The neurological conditions cannot, therefore, explain these findings.

The SDNN is reduced in tachycardia, and the slightly faster heart rate in group 1 relative to group 2 may contribute to the reduction in SDNN in this group. However, there was no difference in heart rates between the subgroups $1 \mathrm{a}$ and $1 \mathrm{~b}$, so this cannot be the complete explanation of the present findings. The sNN50 was not influenced by heart rate.

It is not considered that the use of autonomically active drugs by these patients could account for the abnormalities observed. While a few patients were taking inhaled $\beta$ - agonists, the doses were modest. If these had any effect at all they would be expected to increase HRV (and hence bias the study towards a negative result). Protriptyline has anticholinergic properties, so might have some effect on HRV, although the authors know of no studies of this. However, only four subjects were taking this drug, and if they were excluded from the analysis, it would make no material difference to the results.

The mechanism by which nocturnal hypoxia causes autonomic dysfunction is unclear. Hypoxia has been implicated as a direct cause of peripheral nerve damage in COPD [12]. It is possible that autonomic neurones may be able to survive prolonged periods of mild hypoxia, but that damage occurs following more severe hypoxaemia, even if this is of only a few minutes duration. This could explain why HRV was more closely correlated with the minimum oxygen saturation than with the duration of desaturation or the mean nocturnal oxygen saturation. Whether this damage is reversible or not is unknown, and further studies to investigate the effects of treatment with noninvasive ventilation on autonomic function in patients with nocturnal hypoventilation are required.

The prognostic significance of these findings is unclear. However, in other conditions, autonomic dysfunction as measured by HRV analysis has been shown to be associated with a significant morbidity and mortality. Abnormal HRV is associated with an increased risk of cardiac dysrhythmias [25], and death in conditions including ischaemic heart disease [26], myocardial infarction [27], and chronic heart failure [23]. Many of these subjects had SDNN below $100 \mathrm{~ms}$, a level which is associated with a $17 \%$ 1-yr mortality in patients with cardiac failure [23]. An increased mortality has been demonstrated in hypoxic COPD patients with autonomic dysfunction [10], and it is therefore possible that HRV analysis may provide a tool for prognostic evaluation in patients with nocturnal oxygen desaturation, but this requires further study.

In conclusion, heart rate variability is abnormal in patients with nocturnal oxygen desaturation due to chest wall deformity or neuromuscular disease, and appears to be related to the severity of nocturnal oxygen desaturation. Further studies are needed to clarify whether the abnormal autonomic function is of prognostic significance, and whether it is affected by treatment of hypoventilation.

Acknowledgements. The authors would like to thank S. Evans, R. Riley, and M. Latham for assistance in collecting the data, and J. Corrigan, S. Deakin, J. Lynch, and G. Tinker for performing heart rate variability analyses.

\section{References}

1. Leger P, Bedicam JM, Cornette A, et al. Nasal intermittent positive pressure ventilation. Long-term follow-up in patients with severe chronic respiratory insufficiency. Chest 1994; 105: 100-105.

2. Simonds AK, Elliott MW. Outcome of domiciliary nasal intermittent positive pressure ventilation in restrictive and obstructive disorders. Thorax 1995; 50: 604-609.

3. Sawicka EH, Branthwaite MA. Respiration during sleep in kyphoscoliosis. Thorax 1987; 42: 801-808. 
4. Medical Research Council Working Party. Long-term domiciliary oxygen therapy in chronic hypoxic cor pulmonale complicating chronic bronchitis and emphysema. Lancet 1981; i: 681-686.

5. Nocturnal Oxygen Therapy Trial Group. Continuous or nocturnal oxygen therapy in hypoxemic chronic lung disease: a clinical trial. Ann Intern Med 1980; 93: 391398.

6. Kleiger RE, Lefrak SS, Biggs D, Senior RM. How to detect and treat arrhythmias in chronic lung disease. Geriatrics 1976; 31: 69-75.

7. Stewart AG, Waterhouse JC, Howard P. Cardiovascular autonomic nerve function in patients with hypoxaemic chronic obstructive pulmonary disease. Eur Respir $J$ 1991; 4: 1207-1214.

8. Hjalmarsen A, Aasebo U, Aleksandersen G, Jorde R. Cardiovascular responses to tests for autonomic dysfunction in patients with chronic obstructive pulmonary disease with and without continuous long-term oxygen therapy. $J$ Auton Nerv Syst 1996; 60: 169-174.

9. Stewart AG, Marsh F, Waterhouse JC, Howard P. Autonomic nerve dysfunction in COPD as assessed by the acetylcholine sweat-spot test. Eur Respir $J$ 1994; 7: 1090-1095.

10. Stewart AG, Waterhouse JC, Howard P. The QTc interval, autonomic neuropathy and mortality in hypoxaemic COPD. Respir Med 1995; 89: 79-84.

11. Kinsman RA, Yaroush RA, Fernandez E, Dirks JF, Schocket M, Fukuhara J. Symptoms and experiences in chronic bronchitis and emphysema. Chest 1983; 83: 755761.

12. Gasnault J, Moore N, Arnaud F, Rondot P. Peripheral neuropathies during hypoxaemic chronic obstructive airways disease. Bull Eur Physiopathol Respir 1987; 23: Suppl 11, 199s-202s.

13. Batin PD, Nolan J. Assessment of autonomic function: reflex testing or variability analysis? J Amb Monitoring 1996; 9: 255-273.

14. Nolan J, Flapan AD, Goodfield NE, et al. Measurement of parasympathetic activity from 24-hour ambulatory electrocardiograms and its reproducibility and sensitivity in normal subjects, patients with symptomatic myocardial ischaemia, and patients with diabetes mellitus. $\mathrm{Am} \mathrm{J}$ Cardiol 1996; 77: 154-158.

15. Pagani M, Malfatto G, Pierini S, et al. Spectral analysis of heart rate variability in the assessment of autonomic diabetic neuropathy. J Auton Nerv Syst 1988; 23: 143153.
16. Molgaard H. Evaluation of the Reynolds Pathfinder II system for $24 \mathrm{~h}$ heart rate variability analysis. Eur Heart $J$ 1991; 12: 1153-1162.

17. Neilson JMM. High speed analysis of ventricular arrhythmias from 24 hour recordings. Comput Cardiol 1974; 55: 9.

18. Bjerregaard P. The quality of ambulatory ECG recordings and accuracy of semi automatic arrhythmia analysis. An evaluation of the Medilog-Pathfinder system. Eur Heart $J$ 1980; 1: 417-419.

19. Kleiger RE, Bosner MS, Rottmans JN, Stein PK. Time domain measurements of heart rate variability. $J A m b$ Monitoring 1993; 6: 1-18.

20. Ewing DJ, Neilson JMM, Travis P. New method for assessing cardiac parasympathetic activity using 24 hour electrocardiograms. Br Heart J 1984; 52: 396-402.

21. McAreavey D, Neilson JW, Ewing DJ, Russel DC. Cardiac parasympathetic activity during the early hours of acute myocardial infarction. Br Heart $J$ 1989; 62: 165170.

22. Ewing DJ, Neilson JMM, Shapiro CM, Stewart JA, Reid W. Twenty four hour heart rate variability: effects of posture, sleep, and time of day in healthy controls and comparison with bedside tests of autonomic function in diabetic patients. Br Heart J 1991; 65: 239-244.

23. Nolan J, Batin PD, Andrews R, et al. A prospective study of heart rate variability and mortality in chronic heart failure; results of the United Kingdom heart failure evaluation and assessment of risk trial (UK-HEART). Circulation 1998; 98: 1510-1536.

24. Molgaard H, Hermansen K, Bjerregaard P. Spectral components of short-term RR interval variability in healthy subjects and effects of risk factors. Eur Heart J 1994; 15 : 1174-1183.

25. Farrell TG, Bashir Y, Cripps T, et al. Risk stratification for arrhythmic events in postinfarction patients based on heart rate variability, ambulatory electrocardiographic variables, and the signal averaged electrocardiogram. $J$ Am Coll Cardiol 1991; 18: 687-697.

26. Rich MW, Saini JS, Kleiger RE, Carney RM, te Velde A, Freedland KE. Correlation of heart rate variability with clinical and angiographic variables and late mortality after coronary angiography. Am J Cardiol 1988; 62: 714-717.

27. Kleiger RE, Miller JP, Bigger JT Jr, Moss AJ. Decreased heart rate variability and its association with increased mortality after acute myocardial infarction. Multicentre Post-Infarction Research Group. Am J Cardiol 1987; 59: 256-262. 